\title{
El modelo de responsabilidad personal y social a través del deporte: revisión bibliográfica Personal and social responsibility model through sports: a bibliographic review
}

Bernardino Javier Sánchez-Alcaraz Martínez, Javier Courel Ibáñez, Cristina Sánchez Ramírez, Alfonso Valero Valenzuela, Alberto Gómez Mármol

Universidad de Murcia (España)

Resumen. El Modelo de Responsabilidad Personal y Social (MRPS) ha sido aplicado durante más de 30 años con el objetivo de mejorar los valores de los adolescentes a través de la actividad física y el deporte. El objetivo de este trabajo es realizar una revisión de los diferentes estudios que han implementado el MRPS. Se realizó un proceso de revisión a través del proceso PRISMA. Tras el proceso de identificación y selección, se obtuvieron un total de 35 trabajos. Se analizaron variables como el país de aplicación, la evolución de los estudios a lo largo de los años, la duración del programa, el número de alumnos al que se aplicó, el tipo de variables que evaluaron. Finalmente, se realizó una síntesis y discusión de los principales resultados de los trabajos.

Palabras clave: Deporte, Educación Física, Educación en Valores, Responsabilidad Personal y Social, Revisión bibliográfica.

Abstract. The Model of Personal and Social Responsibility (MRPS) has been applied for 30 years with the aim of improving adolescents' values through physical activity and sport. The aim of this work is to carry out a review of different studies that have implemented the MRPS. A review process was performed using the PRISMA protocol. After the identification and selection process, a total of 35 research articles were obtained. Variables such as country of application, evolution of the studies through the years, duration of the program, number of students, and type of the variables evaluated were analyzed. Finally, a summary and discussion of the main results was presented.

Keywords: Sport, Physical Education, Education in Values, Personal and Social Responsibility, Bibliographic Review.

\section{Introducción}

Los problemas de convivencia entre la población joven suponen un tema de debate actual en el seno de diversos países de la Unión Europea (Cangas, Gázquez, Pérez-Fuentes, Padilla, y Miras, 2007; López, 2004), debido al aumento, en los últimos años, del absentismo escolar, acompañado de un clima de relación social en el que se localizan conductas relacionadas con intimidaciones, maltrato, xenofobia, agresiones o consumo de drogas en los centros escolares (Gázquez, Pérez-Fuentes, Carrión, Santiuste, 2010; Ruiz et al., 2006; Serrano et al., 2018). Entre muchas de las intervenciones con el objeto de abordar esta problemática, las que se llevan a cabo en el contexto de la educación física y la enseñanza deportiva extraescolar han sido recurrentemente objeto de análisis, debido fundamentalmente a diferentes factores: en primer lugar, en estos entornos educativos es más sencillo el acceso a los adolescentes; en segundo lugar, la variedad y popularidad de estas actividades las hacen muy atractivas para gran parte de las personas en edad escolar; y por último, pero no menos importante, las características de estas actividades permiten que los jóvenes puedan establecer relaciones interpersonales muy abiertas con el educador y entre sus iguales (Escartí, Pascual, y Gutiérrez, 2005; Rubio-Rodríguez, Serna, Hernández y Varón, 2019).

Sin embargo, el deporte y la educación física por si mismos no disminuyen las conductas citadas anteriormente de forma automática (Carranza y Mora, 2003), por lo que los cambios en los valores y las actitudes ocurren con más facilidad si son planteados de un modo sistemático y si tienen una orientación educativa en el propio contexto deportivo, ya que a pesar de que éstos se desarrollan en ambientes atractivos e interactivos, emocionantes y divertidos para los

Fecha recepción: 10-10-18. Fecha de aceptación: 01-04-19 Bernardino Javier Sánchez-Alcaraz Martínez anaburruezo@hotmail.com jóvenes, sin la orientación pedagógica adecuada también pueden suponer en ocasiones entornos poco educativos (Cecchini, Montero, y Peña, 2003; Jaqueira, Lavega, Lagardera, Aráujo y Rodrigues, 2014; Sánchez-Alcaraz, Bejerano-Urrea, Valero-Valenzuela, Gómez-Mármol., y CourelIbáñez, 2018). De este modo, un programa físico-deportivo debería estar bien estructurado para que pueda constituir un instrumento efectivo de promoción de la responsabilidad personal y social y que esta forma favorezca la convivencia aprovechando la alta interactividad y el carácter emocional de la actividad deportiva que permite enseñar valores y promover el desarrollo social y emocional (Courel-Ibáñez, Sánchez-Alcaraz, Gómez-Mármol, Valero-Valenzuela y Moreno-Murcia, 2019; Martinek, Schilling, y Johnson, 2001).

Uno de los modelos que se ha mostrado más efectivo ha sido El Modelo de Responsabilidad Personal y Social (MRPS), desarrollado por Donald Hellison, hace más de treinta años. Aunque en sus inicios este fue concebido para trabajar los valores a través de la actividad física y el deporte con jóvenes socialmente desfavorecidos, en los últimos años este Modelo ha sido utilizado para el desarrollo de aspectos psicosociales tales como respeto, autocontrol autoestima, empatía, esfuerzo, autonomía, cooperación, ayuda a los demás y liderazgo en otros contextos deportivos como las actividades extraescolares o entrenamientos y competiciones (Pardo y García-Arjona, 2011; Sánchez-Alcaraz et al., 2018). En el Programa, los participantes aprenden a desarrollar su responsabilidad personal y social de modo gradual, a partir de metas concretas y sencillas. Para aprender los comportamientos relacionados con los valores del programa e interiorizar la filosofía del mismo, van aprendiendo por niveles comportamientos y actitudes que les ayudan a convertirse en personas responsables.

Hellison (2011), propone cinco niveles de responsabilidad (Tabla 1), que se presentan a los estudiantes de modo progresivo y acumulativo y que define comportamientos, actitudes y valores de responsabilidad personal y social para 
ser desarrollados a través de la actividad física y el deporte a partir de metas concretas y sencillas (Escartí et al., 2005). Junto a cada nivel, existe una serie de estrategias y métodos específicos a desarrollar por los estudiantes y el profesor, y así poder alcanzar los objetivos que se plantean dentro de cada uno de los niveles del Modelo de Responsabilidad Personal y Social. Siguiendo a Hellison (2011), el propósito de todas las estrategias es mantener a los jóvenes interactuando con los niveles, no sólo para mejorar sus disciplina y motivación en el momento presente, sino lo que es más importante, que las actitudes y comportamientos desarrollados por ellos mismos se transfieran a otras áreas del gimnasio, siendo éste el objetivo último y también el más importante.

Tabla 1.

Niveles del Modelo de Responsabilide Personaly Socia Niveles del Modelo de Responsabilidad Personal y Socia Niveles del Modelo de Responsabilidad Personal y Social Nivel I: Respeto por los derechos y sentimientos de los demás. En este nivel, el educador debe crear en el aula o en el gimnasio una atmósfera física y psicológicamente segura donde los participantes no se encuentren intimidados o amenazados por nadie, donde cada cual pueda manifestarse libremente sin temor a ser menospreciado o burlado (Escartí et al., 2005).

El objetivo de este nivel es ofrecer experiencias positivas a los educandos que participan en el Modelo para potenciar su implicación en el mismo y contribuir a que adquieran una visión positiva de la actividad física y del deporte que la conviertan en nuevo hábito positivo en sus vidas (Jiménez, 2000). Se trata de promover una participación sin discriminación, donde todo los educandos partan con igualdad de oportunidades, sin que la participación se supedite características de sexo, niveles de habilidad u otros criterios discriminatorios como raza o cultura (Pardo y García-Arjona, 2011).

$$
\text { Nivel III. Autonomía personal: }
$$

El objetivo de este nivel es ofrecer experiencias positivas a los educandos que participan en e Modelo para potenciar su implicación en el mismo y contribuir a que adquieran una visió positiva de la actividad física y del deporte que la conviertan en nuevo hábito positivo en sus vidas (Jiménez, 2000). Se trata de promover una participación sin discriminación, donde todo los educandos partan con igualdad de oportunidades, sin que la participación se supedite a características de sexo, niveles de habilidad u otros criterios discriminatorios como raza o cultura (Pardo y García-Arjona, 2011).

Nivel IV: Ayuda a los demás y liderazgo.

Este nivel se centra en dos importantes aspectos de la Responsabilidad Social: la empatía y el liderazgo. Para el desarrollo de la empatía, los estudiantes deben darse cuenta de que los demás pueden tener un punto de vista diferente del suyo y tienen que aprender a respetarlo, poniéndose en el lugar del otro cuando sea necesario (Pardo, 2008). Al trabajar el liderazgo, el profesor asignará a varios alumnos el estar a cargo de un grupo, para que trabajen de form cohesionada y sea dicho líder, el responsable de tomar las decisiones finales con respecto al grupo. En el nivel 4, se ponen como objetivos conseguir ayudar a otros sin arrogancia y sin dogmatismo, ayudar sólo si otros precisan o quieren ayuda, tratando de evitar los comportamientos egocéntricos en los educandos y fomentar sus comportamientos solidarios. $\frac{\text { comportamientos egocéntricos en los educandos y fomentar sus comportamientos solidarios. }}{\text { Nivel V: Fuera del contexto deportivo. }}$ lases, en el patio del recro, en su casa etc) lo que han aprendido en los cuatro nives de programa (Escartí et al., 2005). Los estudiantes que se encuentran en este Nivel muestran respeto, esfuerzo, autonomía y liderazgo no solo en el gimnasio, sino también fuera de é. vidos (Pardo, 2008).

Además, durante el desarrollo del MRPS, las sesiones siguen una misma estructura con el objetivo principal de que los estudiantes puedan acostumbrarse a cumplir unas normas y que sepan exactamente que se espera de ellos. De este modo, como se observa en la tabla 2, la sesión se divide en 4 partes principales (Hellison, 2011). Es importante tener en cuenta lo imprescindible de respetar el formato de la sesión, ya que los alumnos progresarán más rápidamente cuando se les introducen rutinas bien estructuradas que ellos conocen y en las que se sienten más cómodos.

La aplicación de este modelo ha tenido resultados muy positivos en la mejora de los valores en los adolescentes (Pardo y García-Arjona, 2011; Sánchez-Alcaraz, López-Jaime, Valero-Valenzuela, y Gómez-Mármol, 2017). Sin embargo, han existido diferencias metodológicas en su implementación, y no existe un consenso claro de los investigadores sobre algunos aspectos como la duración del tratamiento, el tipo de contenido utilizado, el número de alumnos, etc. (ParedesGarcía, Gómez-Mármol y Sánchez-Alcaraz, 2016). Estas diferencias han sido contrastadas en la reciente revisión sistemática realizada por Pozo, Grao-Cruces, y Pérez-Ordás, (2018),
Tabla 2.

Estructura de la sesión del Modelo de Responsabilidad Personal y Socia Estructura de la sesión del Modelo de Responsabilidad Personal y Socia

Parte 1. Toma de conciencia Durante los primeros 5 minutos el profesor expone los comportamientos que deben aprender practicar ese día, en concordancia al Nivel de Responsabilidad que están trabajando. Una vez que los alumos terminan de entrar encl aula y se sientan en círculo, el profesor expondrá de torma mu breve el objetivo edrcativo del dra, de for comportamientos requeridos y los que no se van a consentir. A continuación, se explicará la actividad física a realizar y las normas para llevarlas a cabo

Parte 2. La responsabilidad en acción diferentes actividades físicas propuestas de acuerdo a los objetivos planteados, y cuyo objetivo es que los estudiantes aprendan a comportarse con responsabilidad realizando los ejercicio planteados por el profesor. Se deben planificar las actividades como vehículo para enseñar la Responsabilidad Personal y Social, por lo que cualquier actividad puede ser útil, siempre que sea motivadora y tenga un significado de aprendizaje para los alumnos.

$\frac{\text { Parte 3. Encuentro de grupo }}{\text { Al finalizar la actividad física, los alumnos se sientan en círculo alrededor del profesor } y}$ dedican un tiempo a compartir ideas, opiniones y pensamientos que han surgido durante la clase. Durante encuentro de grupo, que tiene una duración aproximada de 10 minutos, el profesor lanzará una pregunta abierta orientada a preguntar a los educandos sobre cómo ha funcionado la actividad del día, qué han aprendido, si se han cumplido los objetivos, etc. La misión del profesor en esta fase es conducir la dinámica del grupo haciendo preguntas y dejando tiempo suficiente para que los alumnos contesten, elaborando el profesor finalmente una pequeña conclusión sobre cómo ha ido la sesión y el modo de solucionar los problemas.

Para concluir la sesión, los alumnos, sentados en círculo, durante dos o tres minutos, valora sobre su comportamiento en clase, en relación con el nivel del programa trabajado ese día. También evalúan el comportamiento de sus compañeros y del profesor. Esta evaluación se realiza con un gesto de mano del siguiente modo: el dedo pulgar hacia arriba indica una evaluación positiva, el dedo pulgar hacia el lado indica una evaluación media y el dedo pulgar hacia abajo una evaluación negativa.

en las que se han analizado 22 artículos que han aplicado el MRPS concluyendo que se hace necesario la realización de estudios con una implementación más larga del MRPS, diseños metodológicos de corte cuantitativo y muestras más amplias. Por lo tanto, con el objetivo de seguir profundizando en la aplicación del MRPS, este estudio se propone realizar una revisión de los diferentes estudios que han implementado el Modelo de Responsabilidad Personal y Social, analizando diferentes aspectos como la evolución del número de publicaciones, el tipo de muestra a la que se aplicó el estudio, las variables estudiadas, la duración de la implementación del programa, los contenidos utilizados y los principales resultados obtenidos.

\section{Método}

\section{Diseño del estudio}

Se realizó un proceso de revisión siguiendo los principios de Cartwright-Hatton, Roberts, Chitsabesan, Fothergill, y Harrington (2004); y Webster y Watson (2002). La búsqueda blibliográfica se realizó en tres bases de datos electrónicas (Web of Science, Scopus, and SPORTDiscus) usando las siguientes palabras clave: Modelo de Responsabilidad Y («personal y social» $O$ «Hellison» $O$ «implementación» $O$ «aplicación» $\mathrm{O}$ «Programa» $\mathrm{O}$ «Valores»), Personal and Social AND («Responsibility Model» OR «Hellison» OR «implementation» OR «aplication» OR «Program» OR «values»). La última búsqueda se realizó el 1 de marzo de 2017.

Los criterios de inclusión fueron: (a) estudios que aplicasen el Modelo de Responsabilidad Personal y Social, (b) estudios originales, (c) estudios peer-reviewed y (d) estudios que evaluasen grupo control y experimental. Los resúmenes de congresos, tesis doctorales, conferencias y libros no se incluyeron en los resultados. No hubo restricciones relacionadas con el sexo, edad, características de la muestra o tipo de deporte empleado. Los idiomas incluidos fueron inglés y castellano.

La figura 1 presenta un PRISMA (Preferred Reporting Items for Systematic Reviews and Meta-Analyses) a través 
Tabla 3 .

Resumen de los estudios incluidos tas la revisión de las investigaciones.

\begin{tabular}{|c|c|c|c|c|c|c|c|c|c|}
\hline Autores & Año & País & $\begin{array}{c}\text { Edad } \\
\text { sujetos }\end{array}$ & $\begin{array}{c}\text { Duración } \\
\text { estudio }\end{array}$ & $\begin{array}{c}\text { Tipo de } \\
\text { participantes }\end{array}$ & $\mathbf{N}^{\circ}$ alumnos & Tipo Actividad & Instrumentos & Resultados más destacados \\
\hline Collingwood & 1971 & EE.UU. & $\begin{array}{l}\text { Entre } 1518 \\
\text { años }\end{array}$ & 3 semanas & Jóvenes delincuentes & 31 chicos & $\begin{array}{c}\text { Programa } \\
\text { terapéutico }\end{array}$ & Entrevista & $\begin{array}{l}\text { Experiencias de éxito, autorespeto y } \\
\text { responsabilidad. }\end{array}$ \\
\hline DeBusk y Hellison & 1989 & EE.UU & 9 y 10 años & 3 meses & $\begin{array}{l}\text { Jóvenes problemas } \\
\text { conducta }\end{array}$ & 10 alumnos & Voleibol & Entrevistas & Mejoras en el comportamiento. \\
\hline Georgiadis & 1990 & Grecia & $\begin{array}{l}\text { Entre } 14 \text { y } \\
17 \text { años }\end{array}$ & 3 meses & $\begin{array}{c}\text { Jóvenes piso de } \\
\text { acogida }\end{array}$ & 12 alumnos & Baloncesto & - & $\begin{array}{l}\text { Aumento del autocontrol, autonomía y } \\
\text { trabajo en equipo. }\end{array}$ \\
\hline Lifka & 1990 & EE.UU & $\begin{array}{l}\text { Entre } 11 \text { y } \\
13 \text { años }\end{array}$ & 4 meses & $\begin{array}{l}\text { Jóvenes riesgo } \\
\text { exclusión social }\end{array}$ & - & $\begin{array}{l}\text { Actividad física y } \\
\text { voleibol }\end{array}$ & Cuestionario & $\begin{array}{l}\text { Mejoras en el plano cognitivo, la participación, el } \\
\text { esfuerzo, la autonomía y las relaciones } \\
\text { interpersonales. }\end{array}$ \\
\hline Hellison & 1993 & EE.UU & $\begin{array}{l}\text { Entre } 11 \text { y } \\
13 \text { años }\end{array}$ & 6 meses & Jóvenes en riesgo & - & Baloncesto & - & Mejoras en la responsabilidad personal y social. \\
\hline Cutforth & 1997 & EE.UU & $\begin{array}{l}\text { Entre } 11 \text { y } \\
13 \text { años }\end{array}$ & 24 meses & $\begin{array}{l}\text { Jóvenes } \\
\text { afroamericanos }\end{array}$ & Varios grupos & $\begin{array}{l}\text { Actividades } \\
\text { deportivas }\end{array}$ & - & $\begin{array}{l}\text { Mejoras en el comportamiento, } \\
\text { técnicas de comunicación, } \\
\text { relaciones interpersonales, } \\
\text { disminución de expulsiones y abandonos. }\end{array}$ \\
\hline Cutforth y Pucket & 1999 & EE.UU & $\begin{array}{l}\text { Entre } 10 \text { y } \\
13 \text { años }\end{array}$ & 6 semanas & Escolares & 11 escolares & Baloncesto & - & $\begin{array}{l}\text { Mejoras en la autoconfianza, respeto de los } \\
\text { demás, habilidades para resolver } \\
\text { conflictos, habilidades interpersonales y } \\
\text { entusiasmo por aprendizaje. }\end{array}$ \\
\hline $\begin{array}{l}\text { Cecchini,Montero y } \\
\text { Peña }\end{array}$ & 2003 & España & $\begin{array}{c}12 \text { y } 13 \\
\text { años }\end{array}$ & 2 meses & Escolares & 142 alumnos & Futbol-sala & Cuestionarios & $\begin{array}{l}\text { Mejoras de la retroalimentación personal, el } \\
\text { retraso de la recompensa, autocontrol y } \\
\text { deportividad. }\end{array}$ \\
\hline Hellison y Wrihgt & 2003 & EE.UU & $\begin{array}{l}\text { Entre } 10 \text { y } \\
19 \text { años }\end{array}$ & 9 años & $\begin{array}{l}\text { Jóvenes riesgo } \\
\text { exclusión social }\end{array}$ & 78 jóvenes & $\begin{array}{c}\text { Actividades } \\
\text { Deportivas }\end{array}$ & Cuantitativo. Registros. & $\begin{array}{l}\text { Mejoras en la responsabilidad personal y social y } \\
\text { conductas de liderazgo, } \\
\text { transferencia de comportamiento a } \\
\text { otros contextos. }\end{array}$ \\
\hline $\begin{array}{l}\text { Wright, White y } \\
\text { Gaebler-Spira }\end{array}$ & 2004 & EE.UU. & $\begin{array}{c}4-8 \text { y } 9-11 \\
\text { años }\end{array}$ & 13 semanas & $\begin{array}{c}\text { Jóvenes } \\
\text { discapacitados }\end{array}$ & 5 y 7 alumnos & Artes marciales & Lista de control & Mejoras en la responsabilidad personal y Social. \\
\hline Escartí et al. & 2006 & España & 15-16 años & 3 meses & $\begin{array}{l}\text { Alumnos Educación } \\
\text { Física }\end{array}$ & 13 alumnos & $\begin{array}{l}\text { Clases Educación } \\
\text { Física }\end{array}$ & $\begin{array}{c}\text { Observación de } \\
\text { comportamientos en las } \\
\text { sesiones }\end{array}$ & $\begin{array}{l}\text { Reducción de las conductas agresivas y de } \\
\text { interrupción. }\end{array}$ \\
\hline $\begin{array}{l}\text { Newton, Watson, } \\
\text { Kim y Beacham }\end{array}$ & 2006 & EE.UU. & $10-13$ años & 5 semanas & Jóvenes marginados & 130 participantes & $\begin{array}{l}\text { Deportes (golf, } \\
\text { tenis, natación, } \\
\text { beisbol y futbol) }\end{array}$ & $\begin{array}{cl}\text { Cuestionario TEOSQ y } & 1 \\
\text { CSRQ } & 1 \\
\end{array}$ & $\begin{array}{l}\text { Mejoras en la responsabilidad personal y social y } \\
\text { las orientaciones de meta. }\end{array}$ \\
\hline $\begin{array}{c}\begin{array}{c}\text { Cecchini,Montero,Al } \\
\text { onso, Izquierdo y } \\
\text { Contreras }\end{array} \\
\end{array}$ & 2007 & España & 13-14 años & 2 meses & $\begin{array}{l}\text { Alumnos Educación } \\
\text { Física }\end{array}$ & 186 alumnos & Futbol & Cuestionario de fair play. & $\begin{array}{l}\text { Mejoras en la responsabilidad personal y social, } \\
\text { autocontrol y deportividad. }\end{array}$ \\
\hline $\begin{array}{l}\text { Cecchini, González y } \\
\text { Montero }\end{array}$ & 2007 & España & 17-32 años & - & $\begin{array}{c}\text { Deportistas } \\
\text { universitarios de alto } \\
\text { nivel y deportistas } \\
\text { profesionales }\end{array}$ & 131 alumnos & $\begin{array}{c}\text { Fútbol y } \\
\text { baloncesto }\end{array}$ & $\begin{array}{l}\text { Cuestionarios fair play, } \\
\text { participación, } \\
\text { orientaciones de meta y } \\
\text { actitudes. }\end{array}$ & $\begin{array}{l}\text { Aumento del fair play, participación, } \\
\text { orientaciones de meta y actitudes. }\end{array}$ \\
\hline Pardo & 2008 & España & $\begin{array}{c}\text { Entre } 15 \text { y } \\
19 \text { años }\end{array}$ & $\begin{array}{l}10 \text { semanas } \\
\text { (en cada } \\
\text { centro-3) }\end{array}$ & Jóvenes en riesgo & 51 alumnos & $\begin{array}{l}\text { Clases Educación } \\
\text { Física }\end{array}$ & $\begin{array}{c}\text { Notas de campo, } \\
\text { cuestionarios y diarios } \\
\text { escritos por los alumnos y } \\
\text { entrevistas al final } \\
\end{array}$ & $\begin{array}{l}\text { Mejoras en las actitudes de respeto, } \\
\text { participación, esfuerzo y autonomía. }\end{array}$ \\
\hline Wright y Burton & 2008 & EE.UU. & 14.8 años & - & Jóvenes en riesgo & $\begin{array}{l}23 \text { estudiantes } \\
\text { afroamericanos }\end{array}$ & $\begin{array}{l}\text { Clases Educación } \\
\text { Física } \\
\end{array}$ & Entrevistas & $\begin{array}{l}\text { Mejoras en la responsabilidad personal y social } \\
\text { en alumnos de primaria y secundaria. }\end{array}$ \\
\hline Cecchini et al. & 2008 & España & $\begin{array}{c}\text { Entre 13- } \\
16 \text { años }\end{array}$ & 10 semanas & $\begin{array}{l}\text { Alumnos Educación } \\
\text { Física } \\
\end{array}$ & 159 alumnos & Futbol-sala & $\begin{array}{c}\text { Cuestionarios fair play y } \\
\text { autocontrol }\end{array}$ & Aumento del fair play y autocontrol. \\
\hline Cecchini et al. & 2009 & España & $\begin{array}{c}\text { Entre 10- } \\
12 \text { años }\end{array}$ & 3 meses & $\begin{array}{c}\text { Alumnos Educación } \\
\text { Física }\end{array}$ & 160 alumnos & $\begin{array}{c}\text { Clases Educación } \\
\text { Física } \\
\end{array}$ & Cuestionarios & $\begin{array}{l}\text { Mejoras en comportamientos asertivos y } \\
\text { disminución de las conductas agresivas. }\end{array}$ \\
\hline $\begin{array}{l}\text { Escartí, Gutiérrez, } \\
\text { Pascual y Llopis }\end{array}$ & 2010 & España & $\begin{array}{l}\text { Entre 11-12 } \\
\text { años }\end{array}$ & $\begin{array}{c}1 \text { año } \\
\text { académico }\end{array}$ & $\begin{array}{l}\text { Alumnos Educación } \\
\text { Física }\end{array}$ & 42 alumnos & $\begin{array}{l}\text { Clases Educación } \\
\text { Física }\end{array}$ & Entrevistas & $\begin{array}{l}\text { Mejoras en los comportamientos de } \\
\text { responsabilidad y auto eficiencia. }\end{array}$ \\
\hline $\begin{array}{l}\text { Escartí, Gutiérrez, } \\
\text { Pascual y Marín }\end{array}$ & 2010 & España & $\begin{array}{c}\text { Entre } 13- \\
14 \text { años }\end{array}$ & $\begin{array}{c}1 \text { año } \\
\text { académico }\end{array}$ & $\begin{array}{l}\text { Jóvenes riesgo } \\
\text { abandono escolar }\end{array}$ & 30 alumnos & $\begin{array}{l}\text { Clases Educación } \\
\text { Física }\end{array}$ & $\begin{array}{cc}\text { cuantitativo MSPSE Y } \\
\text { cualitativo entrevistas }\end{array}$ & $\begin{array}{l}\text { Mejoras en conductas responsables, } \\
\text { responsabilidad con sus iguales y } \\
\text { auto eficiencia. }\end{array}$ \\
\hline $\begin{array}{l}\text { Pascual, Escartí, } \\
\text { Llopis y Gutiérrez } \\
\end{array}$ & 2011 & España & $\begin{array}{c}\text { Entre 10-11 } \\
\text { años a }\end{array}$ & $\begin{array}{l}2 \text { cursos } \\
\text { académicos }\end{array}$ & $\begin{array}{c}\text { Alumnos Educación } \\
\text { Física } \\
\end{array}$ & 40 alumnos & $\begin{array}{c}\text { Clases Educación } \\
\text { Física } \\
\end{array}$ & Cualitativo (2entrevistas) $\mathrm{I}$ & Mejoras en la responsabilidad personal y social. \\
\hline $\begin{array}{l}\text { Gutiérrez, Escartí y } \\
\text { Pascual }\end{array}$ & 2011 & España & $\begin{array}{c}\text { Entre 8-15 } \\
\text { años }\end{array}$ & & - & 22 alumnos & - & Cuestionarios & $\begin{array}{l}\text { Aumento de empatía, conducta prosocial, } \\
\text { agresividad, autoeficacia y } \\
\text { responsabilidad personal y social. }\end{array}$ \\
\hline $\begin{array}{l}\text { Balderson } \\
\text { y Martin, }\end{array}$ & 2011 & EE.UU & $\begin{array}{l}\text { Entre 6-8 } \\
\text { años }\end{array}$ & - & $\begin{array}{l}\text { Alumnos Educación } \\
\text { Física }\end{array}$ & 145 alumnos & $\begin{array}{l}\text { Bádminton, } \\
\text { baloncesto y } \\
\text { voleibol }\end{array}$ & Cuestionario & $\begin{array}{l}\text { Reducción de los niveles de } \\
\text { comportamientos antisociales } \\
\text { como el bullying. }\end{array}$ \\
\hline $\begin{array}{c}\text { Sánchez-Alcaraz, } \\
\text { Gómez-Mármol, } \\
\text { Valero, De la Cruz y } \\
\text { Esteban } \\
\end{array}$ & 2012 & España & $\begin{array}{l}6^{\circ} \text { Primaria } \\
\text { y } 3^{\circ} \mathrm{ESO}\end{array}$ & 4 meses & $\begin{array}{l}\text { Alumnos Educación } \\
\text { Física }\end{array}$ & 802 alumnos & $\begin{array}{l}\text { Clases Educación } \\
\text { Física }\end{array}$ & Cuestionario KINDL & $\begin{array}{l}\text { Aumento de la responsabilidad personal y social } \\
\text { y en la calidad de vida en los escolares. }\end{array}$ \\
\hline $\begin{array}{l}\text { Wright, Whitley y } \\
\text { Sabolboro }\end{array}$ & 2012 & EE.UU & $\begin{array}{c}\text { Entre 6-10 } \\
\text { años }\end{array}$ & 6 semanas & Niñas marginadas & 10 alumnos & $\begin{array}{l}\text { Actividad Física } \\
\text { extraescolar }\end{array}$ & Entrevistas & $\begin{array}{l}\text { Reducción de las conductas negativas y mejora } \\
\text { de las conductas de respeto. }\end{array}$ \\
\hline Jung y Wright & 2012 & EE.UU & 14 años 2 & 20 sesiones & $\begin{array}{c}\text { Estudiantes } \\
\text { problemáticos }\end{array}$ & tudiantes & $\begin{array}{l}\text { Clases Educación } \\
\text { Física } \\
\end{array}$ & $\begin{array}{c}\text { Entrevistas individuales y } \\
\text { hojas observacionales }\end{array}$ & Mejoras en la responsabilidad personal y social. \\
\hline $\begin{array}{l}\text { Hayden, Baltzell, } \\
\text { Kilty y McCarthy }\end{array}$ & 2012 & EE.UU & - & 15 sesiones & $\begin{array}{l}\text { Alumnos Educación } \\
\text { Física }\end{array}$ & 110 alumnos & $\begin{array}{c}\text { Beisbol, futbol y } \\
\text { baloncesto }\end{array}$ & $\begin{array}{l}\text { Escala con datos } \\
\text { cuantitativos y cualitativos } \\
\text { y entrevista individual. }\end{array}$ & $\begin{array}{l}\text { Aumento de la integración y mejoras en la } \\
\text { relación profesor-alumno y trasferencia. }\end{array}$ \\
\hline $\begin{array}{l}\text { Sánchez-Alcaraz, } \\
\text { Gómez-Marmol, } \\
\text { Valero y De la Cruz }\end{array}$ & 2013 & España & $\begin{array}{l}6^{\circ} \text { Primaria } \\
\text { y } 3^{\circ} \text { ESO }\end{array}$ & 3 meses & $\begin{array}{l}\text { Alumnos Educación } \\
\text { Física }\end{array}$ & 186 alumnos & $\begin{array}{c}\text { Clases Educación } \\
\text { Física } \\
\end{array}$ & $\begin{array}{c}\text { Cuestionario } \\
\text { responsabilidad personal y } \\
\text { social }\end{array}$ & $\begin{array}{l}\text { Mejora de la responsabilidad personal y } \\
\text { social en alumnos de primaria y secundaria }\end{array}$ \\
\hline $\begin{array}{l}\text { Caballero, Noguera y } \\
\text { Escartí }\end{array}$ & 2013 & España & $\begin{array}{l}\text { Entre 6-10 } \\
\text { años y 11- } \\
13 \text { años }\end{array}$ & - & $\begin{array}{l}\text { Jóvenes en riesgo y } \\
\text { minorías raciales }\end{array}$ & - & - & - & \\
\hline $\begin{array}{l}\text { Caballero y Delgado- } \\
\text { Noguera }\end{array}$ & 2014 & España & $\begin{array}{l}\text { Entre 11-15 } \\
\text { años USA y } \\
11-12 \text { años } \\
\text { España }\end{array}$ & - & $\begin{array}{l}\text { USA Jóvenes riesgo } \\
\text { y alumnos España }\end{array}$ & - & $\begin{array}{c}\text { USA deportes } \\
\text { tradicionales y } \\
\text { España Educación } \\
\text { formal } \\
\end{array}$ & - & $\begin{array}{l}\text { Aumento del desarrollo personal y social a la } \\
\text { vez que los aprendizajes propios de la actividad } \\
\text { Física. }\end{array}$ \\
\hline Caballero & 2015a & España & $\begin{array}{l}\text { Entre } 16 \text { a } \\
23 \text { años }\end{array}$ & 5 meses & $\begin{array}{l}\text { Alumnos Ciencias } \\
\text { de la Actividad } \\
\text { Física y el Deporte }\end{array}$ & 21 alumnos & $\begin{array}{l}\text { CAFD, guías de } \\
\text { rutas a pie, en } \\
\text { bicicleta y a } \\
\text { caballo } \\
\end{array}$ & Entrevista individual & $\begin{array}{l}\text { Mejora en el desarrollo positivo y de la } \\
\text { percepción del alumnado del aprendizaje. }\end{array}$ \\
\hline Caballero & 2015b & España & $\begin{array}{l}\text { Entre } 16 \text { y } \\
23 \text { años }\end{array}$ & 5 meses & $\begin{array}{l}\text { Alumnos Ciencias } \\
\text { de la Actividad } \\
\text { Física y el Deporte }\end{array}$ & 43 alumnos & - & $\begin{array}{c}\text { Cuestionario } \\
\text { responsabilidad personal y } \\
\text { social y entrevista }\end{array}$ & $\begin{array}{l}\text { Mejora en el desarrollo positivo y de la } \\
\text { percepción del alumnado del aprendizaje. }\end{array}$ \\
\hline Bean y Forneris, & 2015 & EE.UU & $\begin{array}{c}\text { Entre } 11-14 \\
\text { años }\end{array}$ & 30 sesiones & $\begin{array}{l}\text { Alumnos Educación } \\
\text { Física }\end{array}$ & 12 alumnos & $\begin{array}{c}\text { Juegos, deportes y } \\
\text { danza }\end{array}$ & Cuestionario & $\begin{array}{l}\text { Mejora de la responsabilidad personal y } \\
\text { Social. }\end{array}$ \\
\hline $\begin{array}{l}\text { Méndez y } \\
\text { Fernández-Río }\end{array}$ & 2016 & España & $\begin{array}{l}\text { Entre 14-17 } \\
\text { años }\end{array}$ & 16 sesiones & $\begin{array}{c}\text { Estudiantes } \\
\text { problemáticos }\end{array}$ & 143 alumnos & - & $\begin{array}{l}\text { Cuestionario y entrevistas } \\
\text { grupales }\end{array}$ & $\begin{array}{l}\text { Mejoras en la actitud hacia la violencia, } \\
\text { la responsabilidad, las metas de amistad y } \\
\text { necesidades psicológicas básicas. }\end{array}$ \\
\hline $\begin{array}{l}\text { Madrid, Prieto- } \\
\text { Ayuso, Samalot- } \\
\text { Rivera y Gil }\end{array}$ & 2016 & España & $\begin{array}{l}1^{\circ} \text { Educación } \\
\text { Primaria }\end{array}$ & 3 meses & $\begin{array}{l}\text { Alumnos Educación } \\
\text { Física }\end{array}$ & 28 alumnos & $\begin{array}{l}\text { Educación Física } \\
\text { extraescolar }\end{array}$ & $\begin{array}{l}\text { Hoja de anotación } \\
\text { sistemática }\end{array}$ & $\begin{array}{l}\text { Mejoras en el compromiso, Educación para la } \\
\text { salud, competitividad y tolerancia. }\end{array}$ \\
\hline
\end{tabular}


de un diagrama de flujo que resume los principales resultados de la búsqueda bibliográfica. Se encontraron 124 estudios en la búsqueda en bases de datos originales (Scopus ${ }^{\circledR}$ $=32$; Web of Science ${ }^{\circledR}=53$; SportDiscus ${ }^{\circledR}=39$ ). Una vez eliminados los estudios duplicados, se procedió al análisis del título y resumen de los estudios aplicando los criterios de inclusión y exclusión sobre 68 trabajos. Los trabajos no eliminados fueron analizados en una segunda etapa a través de la lectura del texto completo basada en los criterios de inclusión y exclusión. Finalmente, se realizó una búsqueda en las referencias bibliográficas de los estudios incluidos, revisando aquellos posibles trabajos citados que pudiesen ser incluidos en la revisión (Webster y Watson, 2002). Finalmente, un total de 35 estudios fueron incluidos en la revisión, los cuales fueron identificados en la lista de referencias con un asterisco.

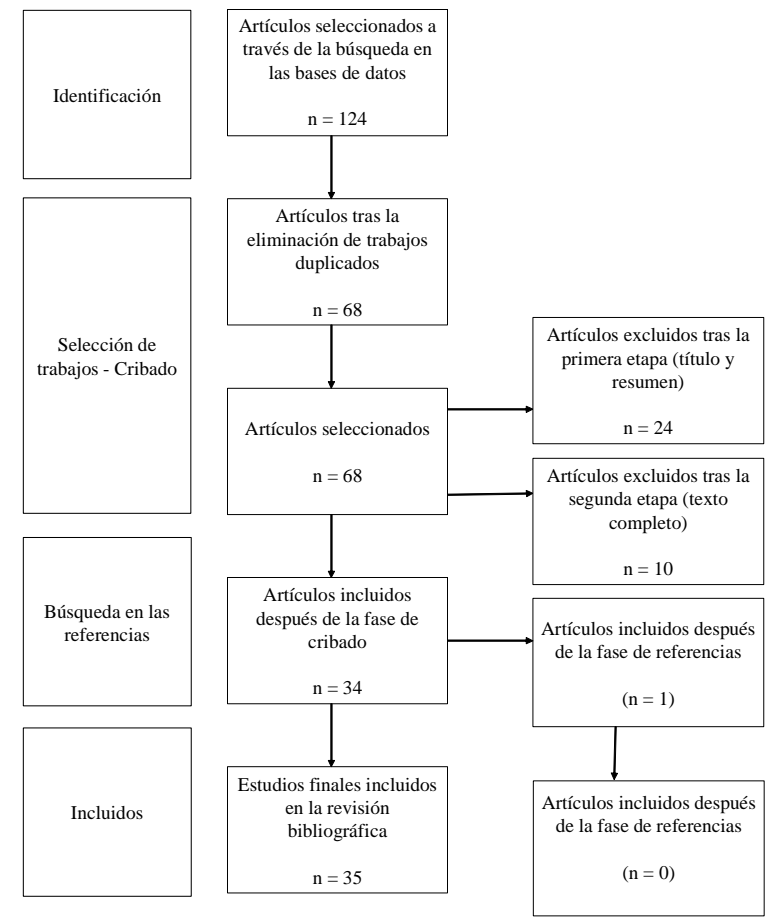

Figura 1. Diagrama de flujo PRISMA mostrando la búsqueda bibliográfica en cada una de las etapas.

\section{Resultados}

La tabla 3 muestra los resultados del análisis sistemático que se ha llevado a cabo con las 35 publicaciones que cumplieron los criterios de inclusión.

Por otro lado, en la Figura 2 se analizó el país en el cual se han desarrollado las investigaciones que han aplicado el MRPS. Como se puede apreciar, la mayoría de investigaciones analizadas se han llevado a cabo en España y Estados Unidos, exceptuando un trabajo realizado en Grecia.

En la Figura 3, se analizó la evolución del número de artículos que han aplicado en MRPS en los últimos años. Como se puede observar en la imagen, desde el año 1985 al año 2005 el número de publicaciones ha sido escaso (6 trabajos), pero a partir de 2006 se produce un crecimiento exponencial en el número de publicaciones (9 entre 2006 y 2010). Además, en estos últimos 6 años ha sido el periodo donde más investigaciones sobre el MRPS se han publicado (14 investigaciones).
Figura 2. Países donde se ha aplicado el MRPS.

Figura 3. Evolución del número de trabajos en los últimos años.

Por otro lado, la tabla 4 muestra la duración en la aplicación del MRPS en los estudios seleccionados. Como se puede observar, el 75,5\% de los programas tiene una duración inferior a 6 meses, mientras que solo el 10,34\% tiene una duración superior a 1 año.

En la tabla 5, se observa el número de alumnos que han participado en las investigaciones. Solo en un 3\% de los estudios han participado menos de 10 alumnos, entre $10 \mathrm{y}$ 100 alumnos en un 50\% de las investigaciones y en un $47 \%$ más de 100 alumnos.

Finalmente, en la Figura 4 se analizó la metodología utilizada en los estudios que han aplicado el MRPS. En este sentido, se puede apreciar una predominanicia de metodologias cuantitativas (cuestionarios, hojas de control, etc), y en un segundo plano estudios que utilizaron metodologías cualitativas (entrevistas) o mixtas (cuantitativas y cualitativas).

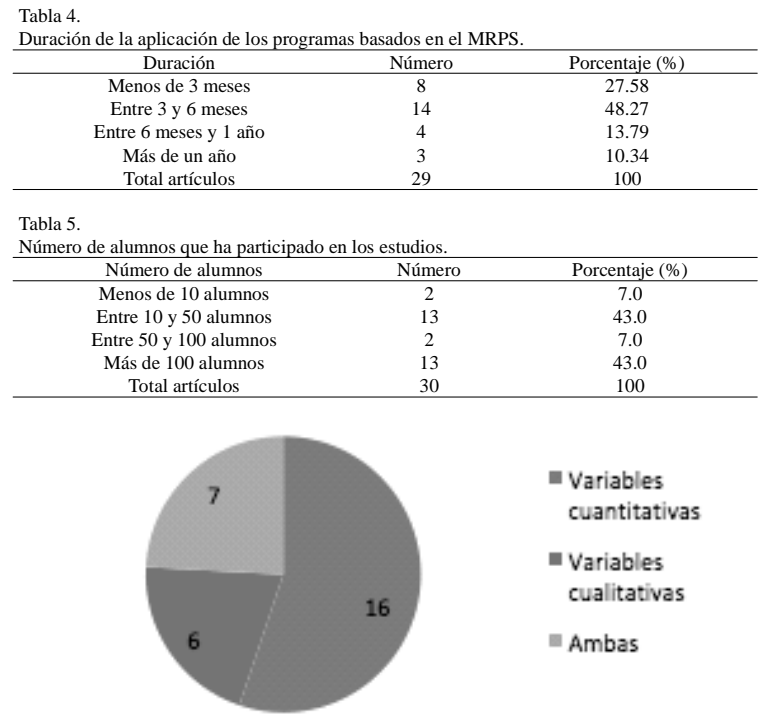

Figura 4. Variables utilizadas en los estudios de aplicación del MRPS 


\section{Discusión}

El objetivo principal de este estudio fue realizar una revisión de los diferentes estudios que han implementado el Modelo de Responsabilidad Personal y Social, analizando diferentes aspectos como la evolución del número de publicaciones, el tipo de muestra a la que se aplicó el estudio, las variables estudiadas, la duración de la implementación del programa, los contenidos utilizados y los principales resultados obtenidos.

\section{Características de la muestra}

Atendiendo a las características de los adolescentes que participaron en los estudios, la mayoría procedían de minorías socialmente desfavorecidas como la Afroamericana (Hellison, 1993; Hellison y Wright, 2003) o la Latina (Cutforth, 1997; Lifka, 1990). Otro de los ámbitos donde se ha trabajado con programas basados en este Modelo, se han centrado en el trabajo con aquellos estudiantes que presentaban problemas de conducta, comportamiento y/o baja motivación hacia el entorno educativo (DeBusk y Hellison, 1989; Newton, Watson, Kim, y Beacham, 2006; Pardo, 2008; Wright y Burton, 2008). Debido a las características que definen a esta población, Hellison (2000) puntualiza que un criterio clave para el éxito de los programas basados en el Modelo de Responsabilidad es mantener el número de participantes reducido, no más de 20 jóvenes. De este modo, generalmente, los estudios aplicados con este modelo mantienen esta premisa, y aunque la muestra del estudio era amplia, los grupos de aplicación del Modelo no sobrepasaban los 20 sujetos (Bean y Forneirs, 2015; Cutforth, 1997; Cutforth y Puckett, 1999; DeBusk y Hellison, 1989; Escartí et al., 2006; Georgiadis, 1990; Hellison y Wright, 2003).

\section{Edad de los participantes}

Con respecto a la edad, la mayoría de los estudios están centrados en niños y adolescentes de entre 6 a 13 años (Cecchini et al., 2003; DeBusk y Hellison, 1989; Hellison, 1993; Lifka, 1990; Sánchez-Alcaraz, et al., 2012; 2013; Wright et al., 2004). Para adolescentes de entre 14 y 17 años, los programas son más bien escasos (Georgiadis, 1990; Escartí et al., 2006). También se han realizado algunos trabajos con sujetos adultos (Caballero, 2015a; 2015b). Sin embargo, hay programas físico-deportivos que combinan diferentes edades como medio para favorecer experiencias de liderazgo entre los participantes de mayor edad (Cutforth, 2000; Cutforth y Puckett, 1999; Hellison y Wright, 2003).

\section{Duración de los programas}

Algunos de los estudios se han llevado a cabo durante un periodo de tiempo relativamente escaso, que oscila entre las 6 a 8 semanas (Cecchini et al., 2003; Cutforth y Puckett, 1999; DeBusk y Hellison, 1989; Newton et al., 2006; Wright et al., 2012), aunque la mayoría de los programas tienen una duración entre trimestral (Madrid, et al., 2016; Sánchez-Alcaraz et al., 2013) y semestral (Cutforth, 1997; Hellison, 1993). Sin embargo, algunos de los estudios aplicaron programas físico-deportivos basados en el Modelo de Responsabilidad durante todo un curso académico (Cutforth, 1997; Escartí et al., 2010; Pascual et al., 2011). Por otro lado, la duración de las sesiones en estos programas fue de aproximadamente una hora por sesión.

\section{Contexto: clases de Educación Física o actividades extraescolares}

La mayoría de las investigaciones han utilizado las clases extraescolares para el desarrollo de sus programas, tanto en instalaciones escolares (Cutforth, 1997; Hellison, 1993) como la universidad (Caballero, 2015a; Cutforth y Puckett, 1999; Hellison y Wright, 2003), Sin embargo, en los últimos años, se ha comenzado a aplicar el Modelo de Responsabilidad Personal en el contexto escolar, utilizando las clases de Educación Física (Escartí et al., 2010; Sánchez-Alcaraz et al., 2012). Sin embargo, siguiendo a Pardo y García-Arjona (2011), Hellison defiende la ventaja de aplicar su Modelo en programas extraescolares debido a que permiten restringir el número de participantes en base a las características del programa y los medios del mismo y la participación de los alumnos es voluntaria. Además, existe una mayor libertad a la hora de planificar horarios y de crear sus propias normas y entorno, ya que proporcionan un lugar seguro alejado de la calle donde los jóvenes pueden ir después de acabar la escuela.

\section{Tipo de deporte realizado en el programa}

Aunque muchos autores han utilizado un único deporte en el desarrollo de sus trabajos, como por ejemplo el baloncesto (Cutforth y Puckett, 1999; Hellison, 1993; Hellison y Wright, 2003) o el fútbol sala (Cecchini et al., 2008), los programas que han sido aplicados en el contexto escolar, utilizando las clases de Educación Física, han aplicado una mezcla de diferentes actividades físicas y deportes (Escartí et al., 2010; Jung y Wright, 2012; Sánchez-Alcaraz et al., 2013). También, en los estudios de Lifka (1990) o DeBusk y Hellison (1989) se emplearon actividades de mantenimiento físico y otros deportes más reglados como el voleibol. De este modo, resulta importante crear un equilibrio entre los deportes más populares dentro de determinados colectivos (fútbol, baloncesto, etc.) y aquellos otros deportes y actividades físicas que aportan nuevas experiencias para los jóvenes, como por ejemplo las actividades en la naturaleza o los juegos cooperativos (Caballero, 2015a).

\section{Resultados de los estudios}

En función de la metodología empleada en los diferentes estudios, podemos dividir entre aquellos que han utilizado metodologías cualitativas (DeBusk y Hellison, 1989; Escartí et al., 2010; Pascual et al., 2011; Wright et al., 2012), y aquellos que han utilizado técnicas cuantitativas como cuestionarios validados, para evaluar los resultados en la aplicación de los diferentes programas (Bean y Forneris, 2015; Cecchini et al., 2007; 2008; 2009; Hellison y Wright; 2003; Madrid et al., 2106; Méndez y Fernández-Río, 2016; Sánchez-Alcaraz et al., 2012; 2013).

La aplicación del MRPS ha mostrado resultados positivos en todos los estudios analizados. En este sentido, algunos trabajos han mostrado como los sujetos desarrollaban mejoras en valores relacionados con la responsabilidad personal como participación y esfuerzo, medidos a través del tiempo de implicación en la tarea, además de mejoras significativas a la hora de trabajar de forma independiente y en 
establecer metas específicas (Georgiadis, 1990; Lifka, 1990). Por otro lado, también se encontraron mejoras en otros valores de la responsabilidad social, tales como ayuda a los demás y respeto, mejorando su autocontrol y el respeto por el material, compañeros, instalaciones, autoridad, normas, etc. (Cecchini et al., 2009; De Busk y Hellison, 1989; Escartí et al., 2010). Otros estudios mostraron mejoras en relación al nivel V (transferencia), a través de mejoras en el comportamiento de los alumnos en el resto de materias y el descenso del número de expulsiones y abandonos (Cutforth, 1997; DeBusk y Hellison, 1989; Georgiadis, 1990).

Además, otros trabajos han encontrado mejoras en otras variables relacionadas con la responsabilidad, como la calidad de vida (Sánchez-Alcaraz et al., 2012), la reducción de comportamientos violentos (Sánchez-Alcaraz et al., 2014) y la mejora de la deportividad (Gómez-Mármol, et al., 2016) En relación a otras habilidades sociales, los estudios de Cutforth (1997) ) y Lifka (1990), mostraron evidencias consistentes de mejora en las técnicas de comunicación y en las relaciones interpersonales de los participantes. El trabajo en equipo y la cooperación son importantes valores para favorecer el desarrollo de la responsabilidad social. En el estudio de Georgiadis (1990), se halló que los participantes mejoraron su habilidad para trabajar en equipo.

Este estudio presenta una serie de limitaciones que deben ser tomadas en cuenta a la hora de interpretar los resultados. En primer lugar, no se ha calculado el tamaño del efecto de los artículos que informaban de estadísticas inferenciales, por lo que futuros trabajos que realicen una revisión sistemática de esta temática podrían incluir estos datos en sus resultados. Además, no se han analizado otras investigaciones que han apostado por la aplicación de una hibridación del MRPS junto con otros modelos de enseñanza como el aprendizaje cooperativo (Menéndez y FernándezRío, 2016; Fernández-Río, y Méndez-Giménez, 2016), por lo que sería interesante tener en cuenta estos trabajos en futuras revisiones. Finalmente, estudios transversales han mostrado relaciones entre el desarrollo de la responsabilidad y con un mayor nivel de actividad física en estudiantes (GómezMármol, Sánchez-Alcaraz, De la Cruz, Valero, y GonzálezVillora, 2017), por lo que parece que futuros estudios pueden utilizar este Modelo, no únicamente para desarrollar valores sino también para mejorar los niveles de práctica deportiva.

\section{Conclusiones}

Las principales conclusiones de este estudio son:

a. La mayoría de los estudios analizados han aplicado el MRPS en estudiantes adolecentes, en horario extraescolar $\mathrm{y}$ en grupos reducidos.

b. Con respecto a los contenidos, la mayoría de los programas han utilizado varios deportes al aplicar el MRPS, y aquellos contenidos relacionados con las clases de EF.

c. En cuanto a la metodología utilizada, la mayoría de estudios analizados utilizan metodologías cuantitativas, como cuestionarios validados.

d. Los resultados de estos estudios revelan que el Modelo de Responsabilidad impactó positivamente en valores de los participantes tales como el respeto, autocontrol, autoestima, empatía, esfuerzo, autonomía, cooperación, ayuda a los demás y liderazgo. Además, se mejoraron otras variables relacionadas con la responsabilidad como la deportividad o la calidad de vida.

\section{Referencias}

Balderson, D.W., y Martin, M. (2011). The Efficacy of the Personal and Social Responsibility Model in a Physical Education Setting. Phenex Journal, 3(3).

Bean, C.N., y Forneirs, T. (2015). Using a time-series analysis to evaluate a female youth-driven physical activity-based life skills program based on the Teaching Personal and Social Responsibility Model. Ágora para la educación física y el deporte, 17(2), 94-114.

Caballero, P. (2015a). Diseño, implementación y evaluación de un programa de actividades en la naturaleza para promover la responsabilidad personal y social en alumnos de formación profesional. Cuadernos de Psicología del Deporte, 15(2), 179-194.

Caballero, P. (2015b). Percepción del alumnado de formación profesional sobre los efectos de un programa de desarrollo positivo (Modelo de Responsabilidad de Hellison). Journal of Sport and Health Research, 7(2), 113-126.

Caballero, P., y Delgado-Noguera, M.A. (2014). Diseño de un programa de desarrollo positivo a través de la actividad física en el medio natural. Journal of Sport and Health Research, 6(1), 29-46.

Caballero, P., Delgado-Noguera, M.A., y Escartí, A. (2013). Analysis of Teaching Personal and Social Responsibility model-based programmes applied in USA and Spain. Journal of Human Sport \& Exercise, 8(2), 427-441.

Cangas, A., Gázquez, J., Pérez-Fuentes, M. C., Padilla, D., y Miras, F. (2007). Evaluación de la violencia escolar y su afectación personal en una muestra de escolares europeos. Psicothema, 19(1), 114-119.

Carranza, M., y Mora, J. M. (2003). Educación física y valores: educando en un mundo complejo. Barcelona: Grao.

Cartwright-Hatton, S., Roberts, C., Chitsabesan, P., Fothergill, C., y Harrington, R. (2004). Systematic review of the efficacy of cognitive behaviour therapies for childhood and adolescent anxiety disorders. British Journal of Clinical Psychology, 43(4), 421-436.

Cecchini, J.A., Fernández, J., y González, C. (2008). Repercusiones del Programa Delfos de educación en valores a través del deporte en jóvenes escolares. Revista de Educación, 346, 167-186.

Cecchini, J.A., González, C. y Montero, J. (2007). Participación en el deporte y fair play. Psicothema, 19(1), 57-64.

Cecchini, J.A., González, C., Alonso, C., Barreal, J.M., Fernández, C., García, M., Llanera, R. y Nuño, P. (2009). Repercusiones del Programa Delfos sobre los niveles de agresividad en el deporte y otros contextos de la vida diaria. Apunts, 96, 34-41.

Cecchini, J.A., Montero, J. y Peña, J.V. (2003). Repercusiones del programa de intervención para desarrollar la responsabilidad personal y social de Hellison sobre los comportamientos de fair-play y el auto-control. Psicothema, 15, 631-637.

Cecchini, J.A., Montero, J., Alonso, A., Izquierdo, M. y Contreras, O. (2007). Effects of Personal and Social 
Responsibility on fair play in sports and self-control in school-aged youths. European Journal of Sport Science, 7(4), 203-211.

Collingwood, T.R. (1971). Survival camping: a therapeutic for rehabilitating problem youth. Arkansas: Arkansas Rehabilitation, Research and Training Center.

Collingwood, T.R. (1997). Providing physical fitness programs to at-risk youth. Quest, 49, 67-84.

Compagnone, N. (1995). Teaching responsibility to rural elementary youth: Going beyond the at-risk boundaries. Journal of Physical Education, Recreation, and Dance, 66(6), 58-63.

Courel-Ibáñez, J., Sánchez-Alcaraz, B.J., Gómez-Mármol, A., Valero-Valenzuela, A., y Moreno-Murcia, J.A. (2019). The moderating role of sportsmanship and violent attitudes on social and personal responsibility in adolescents. A clustering-classification approach. PLoS ONE, 14(2), e0211933.

Cutforth, N. (1997). What's worth doing: Reflections on an after-school program in a Denver elementary school. Quest, 49, 130-139.

Cutforth, N. (2000). Connecting school physical education to the community through service-learning. Journal of Physical Education, Recreation, and Dance, 71(2), 3945.

Cutforth, N. y Pucket, K.M. (1999). An investigation into the organization, challenges, and impact of an urban apprentice teacher program. The Urban Review, 31(2), 153-172.

DeBusk, M. y Hellison, D. (1989). Implementing a Physical Education Self-Responsibility Model for DelinquencyProne Youth. Journal of Teaching in Physical Education, 8, 104-112.

Escartí, A., Gutiérrez, M., Pascual, C. y Llopis, R. (2010). Implementation of the Personal and Social Responsibity Model to improve self-efficacy during physical education classes for primary school children. International Journal of Psychology and Phychological Therapy, 10(3), 387-402.

Escartí, A., Gutiérrez, M., Pascual, C. y Marín, D. (2010). Application of Hellison's Teaching Personal and Social Responsibility Model in Physical Education to improve self-efficacy for adolescents at risk of dropping out of school. The Spanish Journal of Psychology, 13(2), 667.

Escartí, A., Gutiérrez, M., Pascual, C., Marín, D., Martínez, C. y Chacón, Y. (2006). Enseñando responsabilidad personal y social a un grupo de adolescentes de riesgo: un estudio «observacional». Revista de Educación, 341, 373-396.

Escartí, A., Pascual, C. y Gutiérrez, M. (2005). Responsabilidad Personal y Social a través de la Educación física y el deporte. Barcelona: Graó.

Fernández-Río, J., y Méndez-Giménez, A. (2016). ElAprendizaje cooperativo. Modelo Pedagógico para la Educación Física. Retos, 29, 201-206.

Galvan, C. (2004). Investigating the impact of a servicelearning course of teacher candidates and underserved youth. Unpublished doctoral dissertation: University of Northern Colorado, Greeley.

Gázquez, J.J., Pérez-Fuentes, M.C., Carrión, J.J. y Santiuste,
V. (2010). Estudio y análisis de conductas violentas en Educación Secundaria Obligatoria en España. Universitas Psychologica, 9(2), 371-380.

Georgiadis, N. (1990). Does basketball have to be all W's and L's? An alternative program at a residential boys' home. Journal of Physical Education, Recreation and Dance, 61 (6). 42-43.

Gómez-Mármol, A., Sánchez-Alcaraz, B.J., De la Cruz, E., Valero, A., y González-Villora, S. (2017). Personal and social responsibility development through sport participation in youth scholars. Journal of Physical Education and Sport, 17(2), 775-782.

Gómez-Mármol, A., Sánchez-Alcaraz, B.J., Valero, A., y De la Cruz, E. (2016). Desarrollo de la deportividad en escolares de Educación Primaria y Secundaria. E-Balonmano, Revista de Ciencias del Deporte, 11(3), 209-218.

Gutiérrez, M., Escartí, A. y Pascual, C. (2011). Relaciones entre empatía, conducta prosocial, agresividad, autoeficacia y responsabilidad personal y social. Psicothema, 23(1), 13-19.

Hayden, L.A., Baltzell, A., Kilty, K., y McCarthy, J. (2012). Developing responsibility using physical activity: a case study of team support. Ágora para la Educación Física y el Deporte, 4(2), 264-281.

Hellison, D. (1993). The coaching club: Teaching responsibility to inner-city students. Journal of Physical Education, Recreation and Dance, 64(5), 66-70.

Hellison, D. (2011). Teaching responsibility through physical activity ( $3^{\mathrm{a}}$ ed.). Champaign, IL: Human Kinetics.

Hellison, D. y Wright, P.M. (2003). Retention in an urban extended day program: A process-based assessment. Journal of Teaching in Physical Education, 22(4), 369381.

Jaqueira, A.R., Lavega, P., Lagardera, F., Araujo, P. Y Rodrigues, M. (2014). Educando para la paz jugando: género y emociones en la práctica de juegos cooperativos competitivos. Educación Siglo XXI, 32(2), 15-32.

Jiménez, P. (2000). Modelo de intervención para educar en valores a jóvenes en riesgo a través de la actividad física y el deporte. Tesis Doctoral.Universidad Politécnica de Madrid, Madrid, España.

Jung, J. y Wright, P.M. (2012). Application of Helisson's Responsibility Model in South Korea: A Multiple Case Study of 'At-Risk' Middle School Students in Physical Education. Ágora para la Educación Física y el Deporte 14, 140-160.

Kahne, J., Nagaoka, J., Brown, A., O’Brien, J., Quinn, T., y Thiede, K. (2001). Assessing after-school programs as contexts for youth development. Youth and Society, 32(4), 421-446.

Kallusky, J. (2000). In-school programs. In D. Hellison, N. Cutforth, J. Kallusky, T. Martinek, M. Parker y J. Stiehl (Eds.), Youth development and physical activity: Linking universities and communities (pp. 87-114). Champaign, IL: Human Kinetics.

Lifka, B. (1990). Hiding beneath the Stairwell-A dropout prevention program for hispanic youth. Journal of Physical Education, Recreation and Dance, 61 (6), 4041.

López, H. (2004). Padres y alumnos ante el valor de respon- 
sabilidad. Education Siglo XXI, 22, 187-205.

Madrid, P.M., Prieto-Ayuso, A., Samalot-Riviera, A. y Gil, P. (2016). Evaluación de una propuesta extraescolar de conductas apropiadas en educación física y deportiva. $R e$ tos, 30, 36-42.

Martinek, T., Schilling, T., y Johnson, D. (2001). Transfering personal and social responsibility of underserved youth to the classroom. The Urban Review, 33(1), 29-45.

Menéndez, J.I. y Fernández-Rio, J. (2016). Hibridación de los modelos de Educación Deportiva y Responsabilidad Personal y Social: una experiencia a través de un programa de kickboxing educativo. Retos, 30, 150-158.

Newton, M., Watson, D.L., Kim, M. y Beachman, A. (2006). Understanding motivation of underserved youth in physical activity. Youth \& Society, 37(3), 348-371.

Pardo R. (2008). La transmisión de valores a jóvenes socialmente desfavorecidos a través de la actividad física y el deporte. Estudio Múltiple de Casos: Getafe, L'Aquila y Los Ángeles. Tesis Doctoral Europea, Universidad Politécnica de Madrid.

Pardo, R. y García-Arjona, N. (2011). El Modelo de Responsabilidad: desarrollo de aspectos psicosociales en jóvenes socialmente desfavorecidos a través de la actividad física y el deporte. Revista de Psicología y Educación, 6, 211-222.

Paredes-García, I., Gómez-Mármol,A., y Sánchez-Alcaraz, B.J. (2016). El Modelo de Responsabilidad Personal y Social: Estrategias de implementación. Trances, Revista de Transmisión del Conocimiento Educativo y de la Salud, 8(2), 157-174.

Pozo, P., Grao-Cruces, A., y Pérez-Ordas, R. (2018). Teaching personal and social responsibility model-based programmes in physical education: A systematic review. European Physical Education Review,24(1), 56-75. doi:10.1177/1356336X16664749.

Rubio-Rodríguez, G., Serna, H., Hernández, L., y Varón, N. (2019). Validación de un cuestionario para evaluar valores personales y sociales en jóvenes deportistas. Retos, 36, 152-158.

Ruiz, L.M., Rodríguez, P., Martinek, T., Schilling, T., Durán, L.J. y Jiménez, P. (2006). El proyecto esfuerzo: un modelo para el desarrollo de la responsabilidad personal y social a través del deporte. Revista de Educación, 341, 933958.

Sánchez-Alcaraz, B.J., Bejerano-Urrea, A., Valero-Valenzuela, A., Gómez-Mármol, A., y Courel-Ibáñez, J. (2018). Deportividad, disfrute y actitudes hacia la educación física de los estudiantes de educación secundaria. Ágora para la Educación Física y el Deporte, 20(2-3), 319-340.

Sánchez-Alcaraz, B.J., Gómez-Mármol, A., Valero, A., De La Cruz, E., y Díaz, A. (2016). El Modelo de Responsabilidad Personal y Social a través del deporte como propuesta metodológica para la Educación en valores en adolescentes. Espiral. Cuadernos del Profesorado, 9(18), 1626.

Sánchez-Alcaraz, B.J., Gómez-Mármol, A., Valero, A., De la Cruz, E. y Díaz-Suárez, A. (2014). The development of a sport-based personal and social responsibility intervention on daily violence in schools. American Journal of Sport Sciences and Medicine, 2(6A), 13-17.
Sánchez-Alcaraz, B., Gómez-Mármol, A., Valero, A., De La Cruz, E. y Esteban, R. (2012). Influencia del Modelo de Responsabilidad Personal y Social en la Calidad de Vida de los Escolares. Cuadernos de Psicología del Deporte, 12(2), 13-18.

Sánchez-Alcaraz, B.J., Gómez-Mármol, A., Valero-Valenzuela, A., De la Cruz-Sánchez, E., Moreno-Murcia, J.A., y Lochbaum, M.R. (2018). Teachers' pereceptions of personal and social responsibility improvement through a physical education-based intervention. Journal of Physical Education and Sport, 18(4), 2272-2277.

Sánchez-Alcaraz, B.J., Gómez-Mármol, A., Valero, A. y De La Cruz, E. (2013). Aplicación de un programa para la mejora de la responsabilidad personal y social en las clases de Educación Física. Motricidad, European Journal of Human Movement, 30, 121-129.

Sánchez-Alcaraz, B.J., López-Jaime, G, Valero-Valenzuela, A. y Gómez-Mármol, A. (2017). Los programas de educación en valores a través de la educación física y el deporte. Actividad Física y Deporte, Ciencia y Profesión, 28, 45-58.

Serrano, A., Sánchez-Alcaraz, B.J., Courel-Ibáñez, J., GómezMármol, A., y Valero-Valenzuela, A. (2018). Análisis de los niveles de acoso entre iguales y su relación con el nivel de actividad física en educación primaria. Citius, Altius, Fortius, 11(2), 37-48.

Schilling, T. (2001). An investigation of commitment among participants in an extended day physical activity program. Research Quarterly for Exercise and Sport, 72(4), 355365.

Webster, J., y Watson, T. (2002). Analyzing the Past to Prepare for the Future: Writing a Literature Review. MIS Quarterly, 16(2), 13-23.

Wright, P.M., y Burton, S. (2008). Implementation and outcomes of a responsibility-based physical activity program integrated into an intact high school physical education class. Journal of teaching in physical education, 27, 138-154.

Wright, E., Whitley, M. y Sabolboro, G. (2012). Conducting a TPRS program for an underserved girls' summer camp. Ágora para la educación física y el deporte, 14(1), 5-24.

Wright, P.M., White, K. y Gaebler-Spira, D. (2004). Exploring the relevance of the personal and social responsibility model in adapted physical activity: a collective case study. Journal of teaching in physical education, 17(23), 7187.

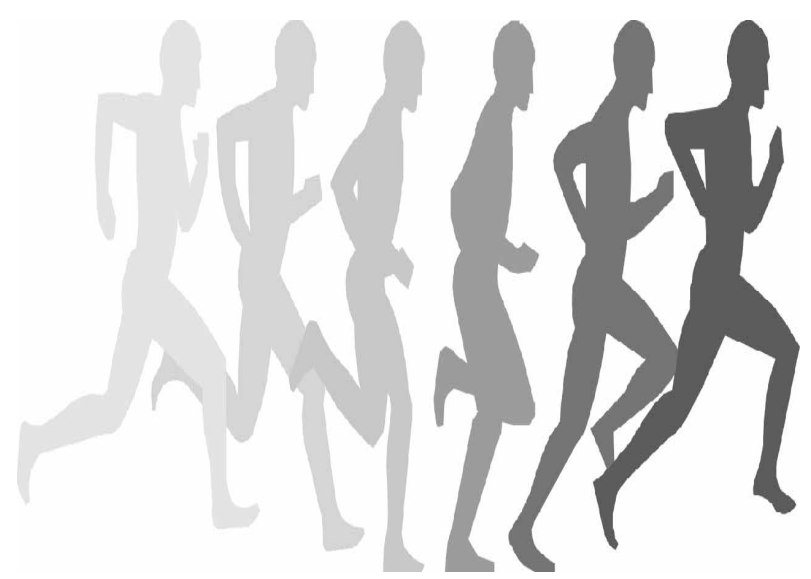

